\title{
Prevalencia de Patrones Antropométricos y Fisiológicos en Población de Adultos Mayores, sobre los 60 Años en Arica, Chile
}

\author{
Prevalence of Anthropometric and Physiological Patterns \\ in the Elderly Population over 60 Years in Arica, Chile
}

"Diaz, J.; "Espinoza-Navarro, O.; ** Rodríguez, H. \& *Moreno, A.

\begin{abstract}
DIAZ, J.; ESPINOZA-NAVARRO, O.; RODRÍGUEZ, H. \& MORENO, A. Prevalencia de patrones antropométricos y fisiológicos en población de adultos mayores, sobre los 60 años en Arica, Chile. Int. J. Morphol., 29(4):1449-1454, 2011.

RESUMEN: El envejecimiento humano es un proceso complejo y multifactorial que involucra aspectos cognitivos, sociales y físicos. El objetivo de este estudio fue determinar valores de algunos patrones antropométricos y fisiológicos y comparar el efecto de la actividad física, de estos patrones en población adulta mayor. Cien adultos mayores en edades a partir de los 60 años participaron de este estudio. En esta población se procedió a medir peso, talla y calcular el índice de masa corporal (IMC), además se obtuvieron registros de la capacidad vital y el consumo máximo de oxígeno. Luego a través de muestra sanguínea se determinó hematocrito y hemoglobina. La población se categorizó en rangos de edades y se separó según género. Posteriormente por encuesta simple de actividades, se separaron en personas activas y sedentarias. Los resultados obtenidos muestran que el IMC en mujeres activas y sedentarias presenta niveles de sobrepeso y obesidad, en varones activos solo a partir de los 70 años se observan valores de normalidad. El colesterol en damas y varones activos presentan valores bajo los $240 \mathrm{mg} / \mathrm{L}$ y la población sedentaria sobre estos valores. Los registros de hemoglobina son significativamente mayores en población activa $(12,3 \mathrm{mg} / \mathrm{dL}$ a 14,2 mg/dL). Los patrones de espirometría muestran registros muy bajos en esta población en estudio. Se concluye que los patrones antropométricos y fisiológicos de esta población en estudio muestra mejores registros en adultos mayores activos.
\end{abstract}

PALABRAS CLAVE: Envejecimiento; Salud pública; Antropometría; Hemoglobina.

\section{INTRODUCCIÓN}

El concepto de envejecimiento humano representa un proceso muy complejo que no tiene una definición operacional estándar. La mayoría de los estudios sobre el adulto mayor se basan sobre todo en factores cronológicos, sin embargo ya está claro que este es un proceso multifactorial que involucra aspectos funcionales, cognitivos, físicos y sociales (Carrasco et al., 2010).

En la actualidad se ha puesto mayor énfasis en como prevenir y mejorar la calidad de vida de las personas de la tercera edad o adulto mayor, interviniendo a los individuos en su alimentación, actividad física y sobre todo en su reinserción social-familiar (Restrepo et al., 2006; Mussart et al., 2006).

El envejecimiento activo y exitoso es una aspiración humana, es decir envejecer bien, sin dolor o con el mínimo sufrimiento (WHO, 2002). Tres aspectos son fundamenta- les a considerar, en primer lugar el potencial individual, en segundo lugar la salud y educación para preservar la juventud y retardar los efectos del envejecimiento y en tercer lugar la disposición y comportamiento de los adultos mayores para un buen desempeño y optimización, esto último con mejores posibilidades a través de entrenamiento y motivación. El adulto mayor con buena capacidad física, está más apto para reforzar el sentido de bienestar y el vínculo social (Brigueiro, 2005; Rabaglietti et al., 2010).

Proponer valores de referencias ya sea antropométricos o fisiológicos es muy difícil en el adulto mayor, debido a que representa una población muy heterogénea, lo cual afecta y se expresa en su composición corporal, y su estado nutricional (Becerra, 2006). El aumento de la población mayor en el mundo es una tendencia epidemiológica. En Chile el 11,5\% corresponde a este tipo de población y se estima que al 2025, llegue a un 22,1\% (Carrasco et al.).

* Universidad de Tarapacá, Arica, Chile.

${ }^{* *}$ Facultad de Medicina, Universidad de Chile, Santiago, Chile. 
El objetivo de este estudio fue determinar la prevalencia de patrones antropométricos de peso, talla e índice de masa corporal (IMC) y patrones fisiológicos de hematocrito, hemoglobina, colesterol, HDL-colesterol y espirometría en adultos mayores de la ciudad de Arica, sedentarios y activos, a partir de los 60 años de edad.

\section{MATERIAL Y MÉTODO}

Para el estudio de los patrones antropométricos y fisiológicos se evaluaron a 100 adultos mayores de la ciudad de Arica, Chile. 55\% correspondió a mujeres y $45 \%$ a hombres, cuya edades fluctúan entre 60 y 80 años y más.

Los sujetos fueron evaluados y categorizados como activos o sedentarios a través de una encuesta simple de actividades, considerando como persona activa a aquellos que realizan acción corporal intencionada, orientada a satisfacer las necesidades de la vida diaria, laboral, social o lúdica. Se consideró como individuo sedentario a aquellos que no realizan ningún tipo de actividad física intencionada, excepto los requerimientos básicos de la vida cotidiana.

Del total de los individuos el 30\% de las mujeres se consideraron sedentarias y $25 \%$ activas. De los hombres el $28 \%$ sedentario y $17 \%$ hombres activos.

Patrones antropométricos. A todos los adultos mayores participantes se les midió el peso $(\mathrm{kg})$ y la talla $(\mathrm{cm})$ y posteriormente se calculó el índice de masa corporal (IMC), según fórmula P/T2 (WHO, 1999).

Patrones fisiológicos. El Volumen máximo de oxígeno $\left(\mathrm{VO}_{2} \mathrm{max}\right)$, se midió aplicando el test submaximal Canadian Fitness test (Jetté et al., 1976).

La capacidad vital expresada en litros por minuto (L/min), se realizó a través de espirómetro electrónico (Vitalogrph + 6800, USA).

Además a cada sujeto se le extrajo una muestra sanguínea por punción venosa para determinar hematocrito y hemoglobina y luego determinar niveles de colesterol y los lípidos de alta densidad (HDL-colesterol).

Posteriormente los individuos fueron separados en grupos etáreos por edades de entre: 60-64 años, 65-69 años, 70-74 años, 75-79 años y 80 y más años. Este estudio fue aprobado por el Comité de Ética/Bioética de la Universidad de Tarapacá, Chile.
Los datos fueron ingresados a planilla Excell 7,0, para su ordenamiento y depuración. Los valores fueron contrastados utilizando el estadístico de "t de Student". Se agruparon por medias las diferentes variables ocupando el software SPSS versión 15 (2007), con un valor de significancia de $\mathrm{p} \leq 0,05$.

\section{RESULTADOS}

Tabla I muestra que los adultos mayores activos, independiente del género, son de mayor talla que la población sedentaria. Con significancia en las mujeres activas de entre 75 a 79 años y en los hombres activos de entre 70 a 79 años. También se refleja que la población sedentaria en general es más numerosa que la población activa ( $58 \%$ y $42 \%$ respectivamente).

En la tabla II se observa que el peso en mujeres activas es menor que el de las sedentarias, siendo significativo en el grupo etáreo de 75-79 años. Los hombres muestran la misma tendencia, excepto en el grupo etáreo de 75-79 años donde las personas activas son de mayor peso que los sedentarios. El IMC presenta a todas la mujeres activas y sedentarias con niveles de sobre peso y obesidad, excepto el grupo de 75-79 años que presentan mujeres con niveles normales de IMC.

En hombres sedentarios de entre 60 a 74 años presentan niveles de sobrepeso, pero a partir de los 74 años en adelante, presentan niveles de normalidad. En los hombres activos desde los 70 años a los 80 años y más se observan niveles normales de IMC.

La Tabla III muestra que toda la población activa de mujeres y hombres tienen valores normales de colesterol $(<240 \mathrm{mg} / \mathrm{dL})$ y toda la población sedentaria en estudio presenta valores mayores a lo normal. Respecto a los niveles de HDL-c, toda la población activa presenta niveles significativamente más elevados ( $>40 \mathrm{mg} / \mathrm{dL})$, respecto a toda la población sedentaria, independiente del género.

En la Tabla IV, se observa que la población activa tanto en mujeres como en hombres tiene mejores porcentajes de hematocrito respecto a la población sedentaria (39\% a $42 \%$ en mujeres y $40 \%$ a $44 \%$ en hombres. Los niveles de hemoglobina son significativamente mayores en la población activa de mujeres y hombres $(12,3-14,2 \mathrm{mg} / \mathrm{dL})$.

En la Tabla V se puede observar que el $\mathrm{VO}_{2} \max$, calculado según el test submaximal Canadian Fitness test, es significativamente mayor en mujeres y hombres activos, 
DIAZ, J.; ESPINOZA-NAVARRO, O.; RODRÍGUEZ, H. \& MORENO, A. Prevalencia de patrones antropométricos y fisiológicos en población de adultos mayores, sobre los 60 años en Arica, Chile. Int. J. Morphol., 29(4):1449-1454, 2011

Tabla I. Talla de los individuos medidos en $\mathrm{cm}$, separados en damas y varones activos y sedentarios. ( ) Indica el número de individuos en cada grupo etáreo según las edades.

\begin{tabular}{llcccc}
\hline \multirow{2}{*}{ Edad } & \multirow{n}{*}{} & \multicolumn{2}{c}{ Mujeres } & \multicolumn{2}{c}{ Hombres } \\
\cline { 3 - 6 } & & Sedentarias (n) & Activas (n) & Sedentarios (n) & Activos (n) \\
\hline $60-64$ & 20 & $1,53(6)$ & $1,58(6)$ & $1,67(5)$ & $1,7(3)$ \\
$65-69$ & 21 & $1,57(6)$ & $1,54(6)$ & $1,64(5)$ & $1,62(3)$ \\
$70-74$ & 23 & $1,51(6)$ & $1,52(7)$ & $1,64(5)$ & $* 1,72(4)$ \\
$75-79$ & 22 & $1,49(6)$ & $* 1,56(6)$ & $1,61(5)$ & $* 1,69(4)$ \\
$>80$ & 14 & $1,51(6)$ & --- & $1,59(5)$ & $1,58(3)$ \\
Total & 100 & $(30)$ & $(25)$ & $(28)$ & $(17)$ \\
\hline $\mathrm{p} \leq 0,05$. & & & & &
\end{tabular}

Tabla II. Peso en kg y el IMC, de la población en estudio según sexo, edad y activos y sedentarios.

\begin{tabular}{|c|c|c|c|c|c|c|c|c|}
\hline \multirow{3}{*}{ Edad } & \multicolumn{4}{|c|}{ Mujeres } & \multicolumn{4}{|c|}{ Hombres } \\
\hline & \multicolumn{2}{|c|}{ Sedentar ias } & \multicolumn{2}{|c|}{ Activas } & \multicolumn{2}{|c|}{ Sedentarios } & \multicolumn{2}{|c|}{ Activos } \\
\hline & kg & IMC & kg & IMC & kg & IMC & kg & IMC \\
\hline $60-64$ & 68,4 & 29,3 & 67,9 & $27,4,4$ & 84,8 & 29,4 & $* 73,1$ & 26,5 \\
\hline $65-69$ & 71,2 & 28,8 & 59,3 & 25,1 & 71,1 & 26,6 & 67,9 & 25,9 \\
\hline $70-74$ & 68 & 30,1 & 65,4 & 28,6 & 68 & 25,4 & 68,4 & 23,2 \\
\hline $75-79$ & 72,3 & 32,5 & $* 59,1$ & 24,4 & 63,7 & 23,9 & $* 71,5$ & 25 \\
\hline$>80$ & 59,6 & 26,2 & --- & ---- & 62,3 & 24,7 & 56,8 & 22,8 \\
\hline
\end{tabular}

Tabla III. Valores obtenidos en sangre para determinar Colesterol y HDL-colesterol, medidos en mg/dL, según sexo, edad y grupo etáreo, activos y sedentarios.

\begin{tabular}{|c|c|c|c|c|c|c|c|c|}
\hline \multirow{3}{*}{ Edad } & \multicolumn{4}{|c|}{ Mujeres } & \multicolumn{4}{|c|}{ Hombres } \\
\hline & \multicolumn{2}{|c|}{ Sedentarias } & \multicolumn{2}{|c|}{ Activas } & \multicolumn{2}{|c|}{ Sedentarios } & \multicolumn{2}{|c|}{ Activos } \\
\hline & Colesterol & HDL-c & Colesterol & HDL-c & Colesterol & HDL-c & Colesterol & HDL-c \\
\hline $60-64$ & 270 & 37 & $* 245$ & $44 *$ & 399 & 34 & $* 175$ & $50 *$ \\
\hline $65-69$ & 300 & 34 & $* 200$ & $50 *$ & 292 & 30 & $* 185$ & $52 *$ \\
\hline $70-74$ & 290 & 36 & $* 179$ & $43^{*}$ & 300 & 36 & *195 & $64 *$ \\
\hline $75-79$ & 275 & 40 & $* 200$ & $45^{*}$ & 280 & 33 & $* 205$ & 47 \\
\hline$>80$ & 323 & 32 & ---- & ---- & 250 & 40 & $* 220$ & 48 \\
\hline
\end{tabular}

$\mathrm{p} \leq 0,05$.

Tabla IV. Resultados obtenidos en muestra sanguínea para los valores de hematocrito, medido en porcentaje y de hemoglobina en mg/dL, según la edad, sexo, sedentarios y activos.

\begin{tabular}{|c|c|c|c|c|c|c|c|c|}
\hline \multirow{3}{*}{ Edad } & \multicolumn{4}{|c|}{ Mujeres } & \multicolumn{4}{|c|}{ Hombres } \\
\hline & \multicolumn{2}{|c|}{ Sedentarias } & \multicolumn{2}{|c|}{ Activas } & \multicolumn{2}{|c|}{ Sedentarios } & \multicolumn{2}{|c|}{ Activos } \\
\hline & $\begin{array}{c}\text { Hematocrito } \\
(\%)\end{array}$ & $\begin{array}{c}\text { Hemoglobina } \\
(\mathbf{m g} / \mathbf{d L})\end{array}$ & $\begin{array}{c}\text { Hematocrito } \\
(\%)\end{array}$ & $\begin{array}{c}\text { Hemoglobina } \\
(\mathbf{m g} / \mathbf{d L})\end{array}$ & $\begin{array}{c}\text { Hematocrito } \\
(\%)\end{array}$ & $\begin{array}{c}\text { Hemoglobina } \\
(\mathbf{m g} / \mathbf{d L})\end{array}$ & $\begin{array}{c}\text { Hematocrito } \\
(\%)\end{array}$ & $\begin{array}{c}\text { Hemoglobina } \\
(\mathrm{mg} / \mathrm{dL})\end{array}$ \\
\hline $60-64$ & 37 & 12,7 & 42 & $13,7^{*}$ & 41 & 12 & 44 & $13,9^{*}$ \\
\hline $65-69$ & 35 & 11,6 & 40 & $13,3^{*}$ & 42 & 13,1 & 44 & 13,1 \\
\hline $70-74$ & 37 & 13,3 & 39 & 13 & 37 & 11,6 & 44 & $14,2 *$ \\
\hline $75-79$ & 40 & 11,1 & 41 & $13,7 *$ & 38 & 12,2 & 44 & $14 * *$ \\
\hline$>80$ & 35 & 11,1 & ---- & ---- & 35 & 11 & 40 & 12,3 \\
\hline
\end{tabular}

(*) Valores de hemoglobina. $\mathrm{p} \leq 0,05$. 
observándose valores en mujeres activas del grupo de 6064 años de $24,2 \mathrm{ml} / \mathrm{kg} / \mathrm{min}$ y en el grupo de 75 a 79 años valores de $21,7 \mathrm{ml} / \mathrm{kg} / \mathrm{min}$. En hombres activos en iguales grupos los valores son de $28,4 \mathrm{ml} / \mathrm{kg} / \mathrm{min}$ y de $26 \mathrm{ml} / \mathrm{kg} / \mathrm{min}$ respectivamente. Al comparar entre género se observa que la población masculina activos y sedentarios, presentan registros significativamente mayores respecto a la población femenina en estudio.
La capacidad vital en mujeres tanto activas como sedentarias presentan muy bajos registros, no presentando diferencias significativas entre ellas, con el valor más alto en mujeres activas de entre 60 a 64 años (2,32 L/min). En cambio los registros obtenidos en hombres activos presentan diferencias significativamente mayores con respecto a hombres sedentarios, con registros de entre $3,45 \mathrm{~L} / \mathrm{min}$ en población de entre 60 a 64 años y 2,9 L/min en hombres activos de 80 años.

Tabla V. Registros obtenidos en el $\mathrm{VO}_{2}$ max, medidos en $\mathrm{ml} / \mathrm{kg} / \mathrm{min}$ y la capacidad vital, medidos en L/min, de la población en estudio.

\begin{tabular}{|c|c|c|c|c|c|c|c|c|}
\hline \multirow{3}{*}{ Edad } & \multicolumn{4}{|c|}{ Mujeres } & \multicolumn{4}{|c|}{ Hombres } \\
\hline & \multicolumn{2}{|c|}{ Sedentarias } & \multicolumn{2}{|c|}{ Activas } & \multicolumn{2}{|c|}{ Sedentarios } & \multicolumn{2}{|c|}{ Activos } \\
\hline & $\mathrm{VO}_{2} \max$ & C. vital & $\mathrm{VO}_{2} \max$ & C. vital & $\mathrm{VO}_{2} \max$ & C. vital & $\mathrm{VO}_{2} \max ^{x}$ & C. vital \\
\hline $60-64$ & 20,4 & 2,22 & $* 24,2$ & $2,32 *$ & 23,4 & 3,28 & $* 28,4$ & 3,45 \\
\hline $65-69$ & 18 & 2,21 & $* 20$ & $1,88^{*}$ & 24,3 & 2,04 & $* 29,8$ & $2,8^{*}$ \\
\hline $70-74$ & 17 & 1,89 & $* 19,8$ & 1,85 & 22,7 & 1,91 & $* 30,8$ & $3,22 *$ \\
\hline $75-79$ & 14,5 & 1,71 & $* 21,7$ & 1,57 & 19,8 & 2,26 & $* 26$ & $3,0^{*}$ \\
\hline$>80$ & 14,1 & 1,73 & --- & ---- & 20,6 & 2,41 & $* 24,5$ & $2,88^{*}$ \\
\hline
\end{tabular}

$\mathrm{VO}_{2} \max =\mathrm{VO}_{2} \max$ en $\mathrm{ml} / \mathrm{kg} / \mathrm{min} ; \mathrm{C}$. Vital $=$ Capacidad vital en $\mathrm{L} / \mathrm{min} . \mathrm{p} \leq 0,05$.

\section{DISCUSIÓN}

El envejecimiento es un proceso complejo con cambios moleculares, celulares, fisiológicos y psicológicos, magnificado por lo general por una alimentación deficiente en energía, calcio, fósforo, vitamina $\mathrm{A}$, vitamina $\mathrm{C}$ y fibras.

El sedentarismo que acompaña esta etapa de la vida, se expresa en desfavorables niveles de lípidos y altos niveles glucosídicos. El esfuerzo físico controlado, poca tensión emocional, resultan ser muy importantes en los adultos mayores para un mejor equilibrio metabólico (Mussart et al.).

En Chile actualmente un $11,5 \%$ de la población corresponde al grupo de la llamada "tercera edad" o adulto mayor y se espera que para el 2025 llegue a un 20,1\%, un fenómeno que va en aumento, esperando que para el 2050 la población de edad avanzada supere a la población juvenil.

Dos grandes tendencias se han planteado para enfrentar esta problemática, la norteamericana con el postulado del "envejecimiento exitoso" y la europea con el "envejecimiento activo", ambas buscan una vejez más grata. Finalmente la "World Health Organization" (WHO) en el año 2002, adopta el envejecimiento activo (active ageing), como mejor alternativa para esta compleja problemática. Lo anterior es una sólida base para que en Chile se tome conciencia del trato digno al adulto mayor, el cual debería tener una condición de privilegio, sobre todo en los hogares de acogida (Walker, 2002; WHO 2002; Carrasco et al.; Sepúlveda et al., 2010; Ramos et al., 2010; Montejo et al., 2011).
Patrones antropométricos. En nuestro estudio los valores de peso talla y IMC (Tablas I y II), son muy similares a los encontrados en población española, donde el mayor porcentaje de adultos mayores corresponden a mujeres (55\%), de igual forma las mujeres sedentarias muestran mayor índice de masa corporal. Los hombres activos presentan índices de IMC normales. Es importante destacar que la determinación de la estatura en los adultos mayores, es uno de los parámetros más difíciles de determinar, sobre todo debido a problemas de cifosis y pérdida de estatura por compresión de los discos vertebrales, estos valores finalmente inciden en el IMC, es posible entonces que los valores o patrones antropométricos en esta población requiera de un ajuste en los valores de normalidad (Becerra; Méndez et al., 2010; Jiménez et al., 2011).

Patrones fisiológicos. Los niveles de colesterol (Tabla III), son significativamente más altos en los adultos sedentarios, tanto en mujeres como en hombres, sin embargo los adultos activos muestran valores normales. En cuanto al HDLcolesterol toda la población activa en estudio, presentan valores significativamente más elevados que la población sedentaria. Estos resultados concuerdan con los estudios realizados por Viñes et al. (2007).

Los valores de hematocrito y hemoglobina (Tabla IV), muestra que los adultos mayores sedentarios, mujeres y hombres, bajos porcentajes de hematocrito y de hemoglobina, con respecto a la población activa. Estudios realizados por Mías et al. (2003), consideran que la baja de hematocrito y hemoglobina se relaciona íntimamente con factores nutricios y de salud, conclusiones similares a las reportadas en el estudio en población chilena adulta, realizada por Carrasco et al. 
En la Tabla V, se puede observar que la población activa tiene mejores registros en el consumo máximo de oxígeno (VO2 max). El VO2 max suele disminuir aproximadamente un $10 \%$ por cada década. Los autores consideran que por debajo de los $15 \mathrm{ml} / \mathrm{kg}$ x min, se pierde la independencia funcional lo que se expresaría a partir de los 85 años en población sedentaria. Nuestro trabajo registra esos valores en mujeres sedentarias a partir de los 75 años, lo que no se observa en hombres activos y sedentarios (Ocampo \& Gutiérrez, 2005; Zaragoza et al., 2005; Allison, 2007; Ramos et al.).

La capacidad vital también expresa mejores valores en la población activa en estudio (Tabla V). Sin embargo los resultados de este estudio muestran registros muy bajos, según los estándares propuestos por Pérez-Padilla et al. (2006), quienes proponen valores de normalidad de acuerdo a criterios norteamericanos, dando valores de $2,19 \pm 0,65$ para mujeres y de 4,21 $\pm 0,92$ para hombres.

Es innegable que finalmente el adulto mayor entra en un estado de fragilidad, en el cual los parámetros antropométricos y fisiológicos, tienden a un funcionamiento especial o geriátrico, donde además se aumentan los riesgos de comorbilidad (Carrasco et al.; Sepúlveda et al.; Holliday, 2010). En esta población es importante destacar que la estimulación cognitiva motora toma un mayor realce (Karlamangla et al., 2007; Gatica et al., 2010; Ramos et al.; Rey et al., 2011). Todos los autores coinciden que programas de actividad física liviana, educación y mejor socialización, tendrían un impacto positivo en lo social, psicológico y emocional de los adultos mayores (Walker; WHO, 2002; Pérez et al., 2003; Salvà, 2004; Brigueiro; Temple et al., 2008; Harrison et al., 2010).

\section{CONCLUSIONES}

Los resultados obtenidos permiten concluir que los adultos mayores activos, tanto mujeres como hombres, tienen mejores registros antropométricos y fisiológicos, que los adultos mayores sedentarios de esta población chilena en estudio, esto confirmaría las teorías de un envejecimiento activo y exitoso.

Finalmente es importante resaltar que el envejecimiento involucra el pago de pensiones, recarga al sistema de salud, tensiones psicosociales, etc, por lo tanto se hace necesario implementar políticas de Estado y Gobierno que persigan la promoción integral para el envejecimiento activo y exitoso, cuyo objetivo sea la búsqueda de mantener la funcionabilidad y la calidad de vida de esta población, con una activa participación de la sociedad.
AGRADECIMIENTOS. Este trabajo fue financiado por la Universidad de Tarapacá (Arica. Chile) Proyecto de Investigación Mayor UTA N 4710-11.

DIAZ, J.; ESPINOZA-NAVARRO, O.; RODRÍGUEZ, H. \& MORENO, A. Prevalence of anthropometric and physiological patterns in the elderly population, over 60 years in Arica, Chile. Int. J. Morphol., 29(4):1449-1454, 2011.

SUMMARY: The aim of this study was to determine anthropometric and physiological patterns and compare the effect of physical activity in older adults from Arica, Chile. One hundred elderly aged from 60 years on, participated in this study. In this population, we proceeded to measure weight, height and calculated body mass index (BMI) and also obtained records of vital capacity and maximal oxygen consumption. Then through blood samples, hematocrit and haemoglobin were determined. The population was categorized in age ranges and separated by gender. Then by simple questionnaire of activities, they were separated into active and sedentary older adults. The results obtained indicate that the active population has a better record than the sedentary population, both in women as well as in men. When analized according to sex, similar results were observed. Spirometry records of the entire study population are very low. We conclude that physical activity is an important factor in the quality of life of people in the so-called third age, supporting theories of an active and successful aging. The study also reaffirms the behavior of the adult population over 60 years is in reality a complex and multifactorial phenomenon.

KEY WORDS: Elderly; Public Health; Anthropometry; Hemoglobin.

\section{REFERENCIAS BIBLIOGRÁFICAS}

Allison, T. Cardiopulmonary exercise testing. Mayo Clinic Cardiology. $3^{\circ}$ Ed. Rochester, Mayo Clinic Scientific Press, 2007. pp.231-9.

Becerra, F. Tendencias actuales en la valoración antropométrica del anciano. Rev. Fac. Med. Unal, 54(4):283-9, 2006.

Brigueiro, M. Envejecimiento exitoso y tercera edad. Problemas y retos para la promoción de la salud. Invest. Educ. Enferm., 23(1):102-9, 2005.

Carrasco, M.; Martínez, G.; Foradori, A.; Hoyl, T.; Valenzuela, E.; Quiroga, T.; Gac, H.; Ihle, S. \& Marin, P. P. Identificación y caracterización del adulto mayor saludable. Rev. Med. Chil., 138(9):1077-83, 2010.

Harrison, E.; Fisher, K.; Lawson, J.; Chad, K.; Sheppard, S.; Reeder, B.; Ashworth, N. \& Bruner, B. Exploring the role of housing type on physical activity and health status in communitydwelling older adults. Activities, Adaptation \& Aging, 34(2): 98-114. 2010. 
Gatica, R. V.; Elgueta, C. E.; Vidal, S. C.; Cantin, L. M. \& Fuentealba, A. J. Impact of Balance Training with a Virtual Reality in Elderly. Int. J. Morphol., 28(1):303-8, 2010.

Jetté, M.; Campbell, J.; Mongeon, J. \& Routhier, R. The Canadian Home Fitness Test as a predictor for aerobic capacity. Can. Med. Assoc. J., 114(8):680-2, 1976.

Jiménez, M.; Sola, J.; Pérez, C.; Turienzo, M.; Larrañaga, G.; Mancebo, M. et al. Estudio del estado nutricional de los ancianos de Cantabria. Nutr. Hosp., 26(2):345-54, 2011.

Karlamangla, A.; Tinetti, M.; Guralnik, J.; Studenski, S.; Wetle, T. \& Reuben, D. Comorbidity in older adults: nosology of impairment, diseases, and conditions. J. Gerontol. A Biol. Sci. Med. Sci., 62(3):296-300, 2007.

Holliday, R. Aging and the decline in health. Health, 2(6):615-9, 2010.

Méndez, E. E.; Rey, C. M.; Troitiño, A. P.; Menéndez, R. M.; Quintas, L. P. \& Veiga, L. B. Valoración del estado nutricional de pacientes ancianos en Orense. Sociedad Española de Medicina General, 125:61-8, 2010.

Mías, C.; Jurschik, P.; Massoni, T.; Sadurní, M.; Aguilà, J. J.; Solá, R.; Nuin, C. \& Torres, J. Evaluación del estado nutricional de los pacientes mayores atendidos en una unidad de hospitalización a domicilio. Nutr. Hosp., 18(1):6-14, 2003.

Montejo, C.; Toledo, M. \& Corvalán, C. Evaluación y entrenamiento funcional en el adulto mayor. Medwave, 11(2), 2011.

Mussart, N.; Coppo, J. \& Coppo, D. Consecuencias del tipo de actividad laboral sobre algunos indicadores bioquímicos de riesgo aterogénico. Estudio en población geriátrica del nordeste argentino. Acta Bioquím. Clín. Latinoam., 40(1):55-62, 2006.

Ocampo, J. M. \& Gutiérrez, J. Envejecimiento del sistema cardiovascular. Rev. Colomb. Cardiol., 12(2):53-63, 2005.

Pérez, J.; Monroy de Peña, A., Díaz, D.; Flórez, R. Cambios en algunas variables hematológicas en un grupo de mujeres mayores de 55 años luego de un programa de entrenamiento aeróbico. Iatreia, 18(1):283-90, 2003.

Pérez-Padilla, R.; Valdivia, G.; Muiño, A.; López, M. V.; Márquez, M. N.; Montes de Oca, M.; Tálamo, C.; Lisboa, C.; Pertuzé, J.; B Jardim, J. R. \& B Menezes, A. M. Valores de referencia espirométrica en cinco ciudades de Latinoamérica, para sujetos de 40 o más años de edad. Arch. Bronconeumol., 42(7):31725. 2006.

Rabaglietti, E.; Liubicich, M. \& Ciairano, S. Physical and psycological condition of senior people in a residental care facility. The effects of an aerobic training. Health, 2(77):773$80,2010$.
Ramos, C.; Santos, A.; Ochoa, M.; Peña, N.; Ramos, R. \& Ramos, R. Comportamiento fisiológico cardiorespiratorio en el adulto mayor durante el ejercicio físico. Corr. Med. Cient. Holg., 14(3), 2010.

Restrepo, S.; Morales, R.; Ramírez, M.; López, M. \& Varela, L. Los hábitos alimentarios en el adulto mayor y su relación con los procesos protectores y deteriorantes en salud. Rev. Chil. Nutr., 33(3):500-10, 2006.

Rey, A.; Canales, I. \& Táboas, M. Motor-cognitive stimulation of the elderly. Educ. Gerontol., 37(2):138-53, 2011.

Salvà, A. Suma de esfuerzos para la atención a las personas mayores. Rev. Esp. Geriatr. Gerontol., 39(1):6-8, 2004.

Sepúlveda, C.; Rivas, E.; Bustos, L. \& Illesca, M. Perfil sociofamiliar en adultos mayores institucionalizados. Temuco, Padre Las Casas y Nueva Imperial. Cienc. Enferm., 16(3):49-61, 2010.

SPSS Inc. Advanced statistic for windows. Version 15.0, Chicago, USA, 2007.

Temple, B.; Janzen, B. L.; Chad, K.; Bell, G.; Reeder, B. \& Martin, L. The health benefits of a physical activity program for older adults living in congregate housing. Can. J. Public Health, 99(1):36-40, 2008.

Viñez, J.; Díez, J.; Guembe, M.; González, P.; Amézqueta, C.; Barba, J.; Sobejano, I.; Martínez Vila, E.; Grijalba, A. M.; Serrano, M.; Moreno, C.; Los-Arcos, E. \& Guerrero, D. Estudio de riesgo vascular en Navarra: Objetivos y diseño. Prevalencia del síndrome metabólico y de los factores mayores de riesgo vascular. Anales Sis. San. Navarra, 30(1):113-24, 2007.

Walker, A. A strategy for active ageing. Int. Soc. Security Rev., 55(1):121-39, 2002

World Health Organization (WHO). The use and interpretation of anthropometry. Technical report series $N^{\circ} 854$. Geneva, World Health Organization, 1999.

World Health Organization (WHO). Active ageing: A policy framework. Geneva, World Health Organization, 2002.

Zaragoza, J.; Serrano, E. \& Generelo, E. Dimensiones de la condición física saludable: evolución según edad y género. Rev. Int. Med. Cienc. Act. Fís. Deporte, 5(17):50-67, 2005.

Dirección para correspondencia:

Prof. Dr. Omar Espinoza Navarro

Departamento de Biología, Facultad de Ciencias

Universidad de Tarapacá.

Casilla 7/D, General Velásquez 1775, Arica

CHILE

Email: oespinoz@uta.cl

Recibido : 30-08-2011

Aceptado: 27-09-2011 